\title{
CrEOS: Identifying Critical Events in Online Sessions
}

\author{
Meghanath Macha* \\ mmacha@cmu.edu \\ Heinz School, CMU \\ Pittsburgh, Pennsylvania
}

\author{
Shankar Venkitachalam \\ Adobe Inc \\ San Jose \\ svenkita@adobe.com
}

\author{
Deepak Pai \\ Adobe Inc \\ San Jose \\ dpai@adobe.com
}

\begin{abstract}
Analyzing a consumer's experience in an online session is a fundamental task of a marketer to enrich, personalize and enhance their future interactions with a firm. Click (web) or tap (mobile) event streams efficiently capture a consumer's experience in an online session. In this work, we propose CrEOS contributing to the extant literature in the deployment of deep learning techniques to model online consumer behaviour. CrEOS models consumer conversion behaviour using a multi-variable LSTM, enriching the consumer event stream with a series of contextual features. In addition to the predictions, CrEOS identifies events that either hamper or help a conversion at three levels - consumer, consumer segment and transitions in marketing funnel. We validate $\mathrm{CrEOS}$ on click-stream data of a large US based e-commerce firm, compare it with single variable LSTM and discuss the insights derived from the critical event identification considering a series of marketing tasks.
\end{abstract}

\section{CCS CONCEPTS}

- Applied computing $\rightarrow$ Online shopping; • Information systems $\rightarrow$ Web mining.

\section{KEYWORDS}

marketing, consumer behavior, deep learning, counter factual analysis

\section{ACM Reference Format:}

Meghanath Macha, Shankar Venkitachalam, and Deepak Pai. 2020. CrEOS: Identifying Critical Events in Online Sessions. In Companion Proceedings of the Web Conference 2020 (WWW'20 Companion), April 20-24, 2020, Taipei, Taiwan. ACM, New York, NY, USA, 6 pages. https://doi.org/10.1145/3366424. 3382185

\section{INTRODUCTION}

Analyzing a consumer's experience in an online session is a fundamental task of a marketer to enrich, personalize and enhance their future interactions with a firm. Click (web) or tap (mobile) event streams efficiently capture a consumer's experience in an online session [6]. Analysis of click and tap streams to predict a consumer's purchase propensity is an active research area (cf. [5] for a survey). While the earlier works $[3,13]$ relied on extensive feature engineering from the user sessions to predict purchase intent, recently the

*This work was done when Meghanath was an intern at Adobe.

This paper is published under the Creative Commons Attribution 4.0 International (CC-BY 4.0) license. Authors reserve their rights to disseminate the work on their personal and corporate Web sites with the appropriate attribution.

WWW'20 Companion, April 20-24, 2020, Taipei, Taiwan

(c) 2020 IW3C2 (International World Wide Web Conference Committee), published under Creative Commons CC-BY 4.0 License.

ACM ISBN 978-1-4503-7024-0/20/04.

https://doi.org/10.1145/3366424.3382185 trend has shifted to employ end to end deep learning techniques. These primarily involve learning a single variable $[8,11,12,14]$ LSTM where the input is a consumer's event stream to predict the conversion. This recent trend shift reduces the burden on a marketer to perform feature engineering. Moreover, recent research [12] points to evidence that deep learning techniques model consumer conversion better than fixed vector based methods. However, for a marketer, being able to predict a consumer's purchase intent would ideally need to be supplemented by the click or tap events (events hereafter) that have helped a consumer make the purchase decision. While, addition of an attention layer [14] or post-hoc explanations of the conversion behavior have been studied [12], these studies are limited to single variable LSTMs.

Explanations of a conversion model provide useful feedback for a marketer to better enrich the experience of a consumer in the future. For instance, assume a conversion model predicts that a segment of users have a low propensity to convert and supplements the propensity with an explanation that the event "Product Questions" hampers the propensity to purchase. An e-commerce marketer could use this information to hypothesize that the customers are either not satisfied with the answers to the product questions or there is a lack of answers to the consumer's questions provided by the seller. Acting on this information, a marketer could enrich the future experience of a consumer by improving the quality and timeliness of answers on the product pages. Given the usefulness of explanations to a marketer, there is a need to develop techniques that not only predict online conversion but also provide explanations.

In this work, we contribute to the growing literature in the deployment of deep learning techniques $[8,11,12,14]$ to model conversion behaviour in two ways through CrEOS (Critical Events in Online Sessions). First, CrEOS models the conversion behaviour of a consumer using a multi-variable LSTM. This is done by enriching the consumer event stream with other event specific contextual features such as price of product, weekend/weekday to predict conversion. Second, CrEOS provides post hoc explanations of the conversion behaviour at three levels - consumer, consumer segment and marketing funnel [16]. In specific, using CrEOS, a marketer, in addition to estimating the conversion propensity, is able to answer the following three questions.

(1) Consumer - Given a consumer event stream, what events helped or hampered conversion propensity?

(2) Consumer segment - Given a segment of consumer event streams, what events helped or hampered their conversion propensities?

(3) Marketing funnel - Given a segment of consumer event streams, what events helped or hampered their transition from Awareness to Desire in the marketing funnel? 


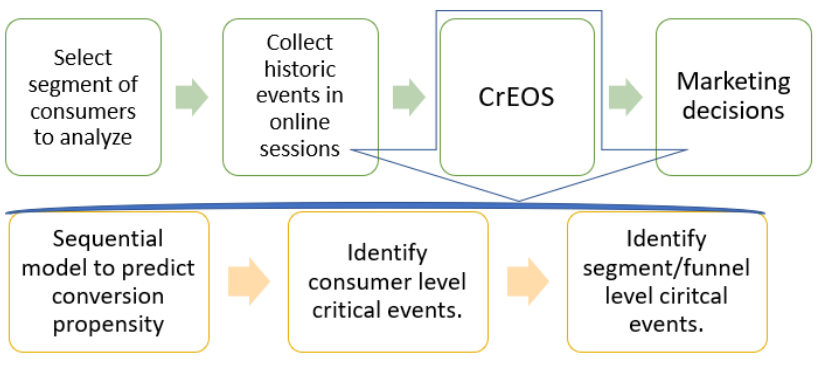

Figure 1: Overview of marketer's workflow in CrEOS.

CrEOS identifies events that either hamper or help the conversion propensity in an event stream (referred as critical events). In the earlier example, "Product Questions" is critical because it hampers the conversion propensity. The events are identified post-hoc through a counter factual analysis that quantifies the importance of each event in the consumer event stream. We extend this to consumer segment and purchase funnel by setting up a hypothesis testing framework. We validate CrEOS on click-stream event data of a large US based e-commerce firm, compare it with single variable LSTM and discuss the insights derived from the critical event identification considering a series of marketing usecases.

\section{METHOD}

\subsection{Marketer's workflow}

In Figure 1, we present the overview of a marketer's workflow in CrEOS. First, a marketer selects a set of consumers to analyze. This is followed by querying the historical online session event stream of the selected consumers. The event stream could comprise of a series of URLs or a specific set of events such as a product home page, review page etc. that a consumer usually performs before making a purchase. While the events could be directly fed into CrEOS, URLs would need to be pre-processed. This could be done by leveraging some of the earlier studies $[3,13]$ that detail categorization of click URLs. Further, each event in the event stream can be augmented with other contextual features such as time of day, weekday/weekend, price of the product etc. The next step is to feed in the historical session information to CrEOS which first learns a multi-variable LSTM to predict the conversion propensity and then produces post-hoc explanations at a consumer, consumer segment and purchase funnel level. This is explained in detail in the next section. Based on the insights gained from the critical events of the selected consumers, a marketer could strategize her marketing decisions accordingly.

\subsection{CrEOS}

2.2.1 Sequential Model to predict conversion propensity. To predict the conversion of a consumer, we learn a one directional multivariable LSTM. Consumer event streams are sequential and of varying lengths, making RNNs a natural model choice for prediction tasks. Further, RNNs provide the flexibility to accommodate other contextual features and often require minimal data processing and feature engineering. Given that consumer historical event streams

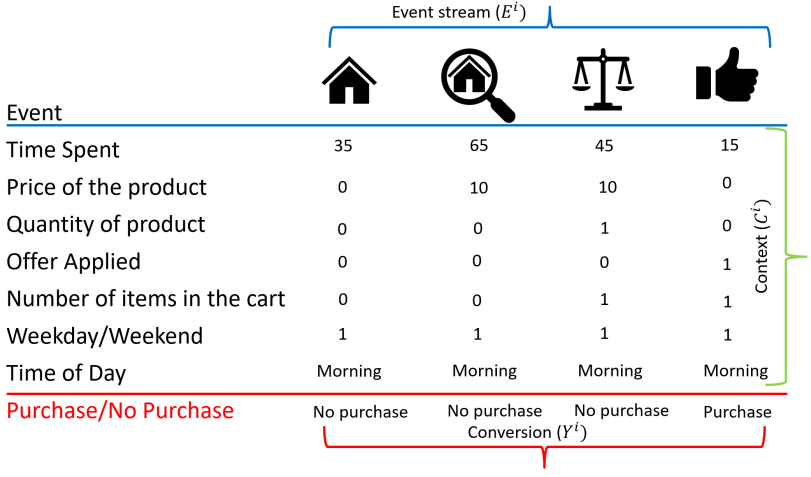

Figure 2: Multi-variable LSTM input and output (red)

can be long, to remedy the vanishing gradients issue and better capture the long term dependencies, we consider LSTMs to model the conversion.

Assume the event sequence for a consumer $i$ is given by $E_{k_{i}}^{i}=$ $\left\{e_{1}^{i}, e_{2}^{i}, e_{3}^{i} \ldots, e_{k_{i}}^{i}\right\}$ where the events are temporally ordered, $k_{i}$ indicates the number of events corresponding to $i^{t h}$ consumer. For an e-commerce website, the events in $E_{k_{i}}^{i}$ usually comprise of product home page, cart addition, review page etc. Let $Y_{k_{i}}^{i}=$ $\left\{y_{1}^{i}, y_{2}^{i}, y_{3}^{i} \ldots, y_{k_{i}}^{i}\right\}$ be a vector of length $k_{i}$ that denotes whether a consumer has made a purchase after each event in $E_{k_{i}}^{i}\left(y_{j}^{i}=1\right.$ if a consumer coverts 0 otherwise). To model $P\left(y_{k_{i}}^{i} \mid E_{k_{i}}^{i}\right)$, the event stream can be further enriched with context based features such as time spent at each event, number of items in the cart during the session, price of the product if the event was a product home page etc.

Formally, consider $C_{k_{i}}^{i}=\left\{\vec{c}_{1}^{i}, \vec{c}_{2}^{i}, \vec{c}_{3}^{i} \ldots, \vec{c}_{k_{i}}^{i}\right\}$, where $\vec{c}_{j}^{i}$ is a vector of these contextual features corresponding to $e_{k_{i}}^{i}$. In CrEOS, we model the conversion problem as $P\left(y_{k_{i}}^{i} \mid\left\{E_{k_{i}}^{i}, C_{k_{i}}^{i}\right\}\right)$. We consider a series of contextual features to augment with the historical event stream $E_{k_{i}}^{i}$. In figure 2, we display an example event stream of Home Page, Product Home, Product Compare, Product Home that end in a purchase. The events are augmented with the seven contextual features considered in CrEOS. We model the $P\left(y_{k_{i}}^{i} \mid\left\{E_{k_{i}}^{i}, C_{k_{i}}^{i}\right\}\right)$ as a binary classification problem minimizing the loss after each event in $E_{k_{i}}^{i}$ and perform a five-fold cross validation to estimate $P\left(y_{k_{i}}^{i} \mid\left\{E_{k_{i}}^{i}, C_{k_{i}}^{i}\right\}\right)$. More details of the training are later discussed in the Section 3.

Next, we introduce our counter factual analysis framework in CrEOS which identifies critical events that contribute to the conversion estimates. We first discuss this at a consumer level and then extend it to a consumer segment and transitions in marketing funnel.

2.2.2 Consumer level critical events. : To identify the critical events that have either hampered or helped a consumer's conversion propensity, we utilize the intermediate estimates of a consumer's conversion propensities $P\left(y_{j}^{i} \mid\left\{E_{j}^{i}, C_{j}^{i}\right\}\right), j \leq k_{i}$. Note that the LSTM model (refer to Figure 2 for input and output structure) to estimate 


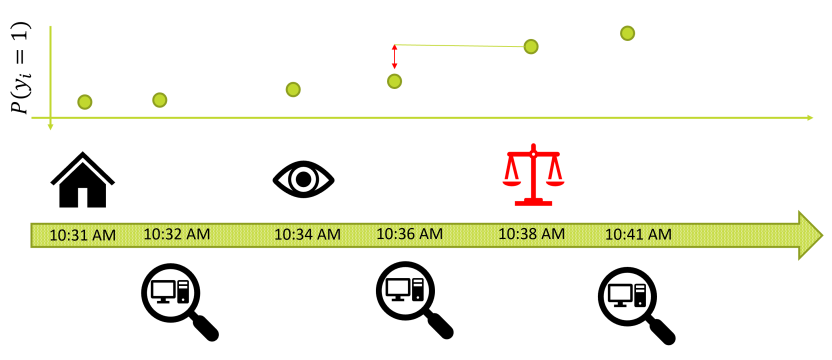

Figure 3: $L\left(e_{j}^{i}\right)$ : Difference in propensities when event is present.

$P\left(y_{k_{i}}^{i} \mid\left\{E_{k_{i}}^{i}, C_{k_{i}}^{i}\right\}\right)$ can be used to estimate conversion propensities for any ordered sub sequence of $\left\{E_{j}^{i}, C_{j}^{i}\right\}, j \leq k_{i}$ of $\left\{E_{k_{i}}^{i}, C_{k_{i}}^{i}\right\}$. We quantify the importance of each event in $e_{j}^{i} \in E_{k_{i}}^{i}$ by using a differences in difference estimate [2] where the event $e_{j}^{i}$ is viewed as a treatment on the consumer's conversion propensity. Drawing an analogy to econometrics, difference in difference estimate computes the effect of a treatment (here $e_{j}^{i}$ ) on an outcome (here consumer's conversion propensity) by comparing the change in the outcome variable for the treatment, compared to the change in outcome variable for the control.

To compute the difference in difference estimate in our setting, we first compute the difference in consumer's conversion propensity at that event $P\left(y_{j}^{i}=1 \mid\left\{E_{j}^{i}, C_{j}^{i}\right\}\right)$ from the prior event $P\left(y_{j-1}^{i}=1 \mid\left\{E_{j-1}^{i}, C_{j-1}^{i}\right\}\right)$ (treatment). This quantifies the influence of $e_{j}^{i}$ on the conversion propensity when the event $e_{j}^{i}$ is present (Figure 3). Next, we consider the counterfactual where the event is absent (control, Figure 4) $\left\{E_{j+1}^{i} \backslash e_{j}^{i}, C_{j+1}^{i} \backslash c_{j}^{i}\right\}$ and recompute the difference in conversion propensity from the prior event. Finally, we perform a difference in the two estimates ${ }^{1}$ to compute the criticality of $e_{j}^{i} \in E_{k_{i}}^{i}, Q\left(e_{j}^{i}\right)$

$$
\begin{gathered}
L\left(e_{j}^{i}\right)=P\left(y_{j}^{i}=1 \mid\left\{E_{j}^{i}, C_{j}^{i}\right\}\right)-P\left(y_{j-1}^{i}=1 \mid\left\{E_{j-1}^{i}, C_{j-1}^{i}\right\}\right) \\
L\left(e_{j}^{i^{*}}\right)=P\left(y_{j+1}^{i}=1 \mid\left\{E_{j+1}^{i} \backslash e_{j}^{i}, C_{j+1}^{i} \backslash c_{j}^{i}\right\}\right)-P\left(y_{j-1}^{i}=1 \mid\left\{E_{j-1}^{i}, C_{j-1}^{i}\right\}\right) \\
Q\left(e_{j}^{i}\right)=L\left(e_{j}^{i}\right)-L\left(e_{j}^{i^{*}}\right)
\end{gathered}
$$

Intuitively, if $Q\left(e_{j}^{i}\right)$ is positive, it would mean the event is critical in increasing the consumer's conversion propensity. On the other hand, if $Q\left(e_{j}^{i}\right)$ is negative, it would mean that the event was detrimental. The estimates of $Q\left(e_{j}^{i}\right)$ can be used by a marketer to infer insights at a consumer level. The estimates could also be used to identify consumer segments relevant to an event. For instance, a marketer could form a consumer segment of consumers who have a positive critical value for the event "Deals Page" on an ecommerce website and personalize their experience by providing better deals in their future visits to improve their likelihood of making a conversion.

\footnotetext{
${ }^{1}$ Note that one cannot use a bi-directional LSTM to learn $P\left(y_{j}^{i} \mid\left\{E_{j}^{i}, C_{j}^{i}\right\}\right)$ to quantify the criticality with our estimate $Q\left(e_{j}^{i}\right)$ since $L\left(e_{j}^{i}\right), L\left(e_{j}^{i^{*}}\right.$ would no longer capture the difference in propensity when the event is present and the counterfactual.
}

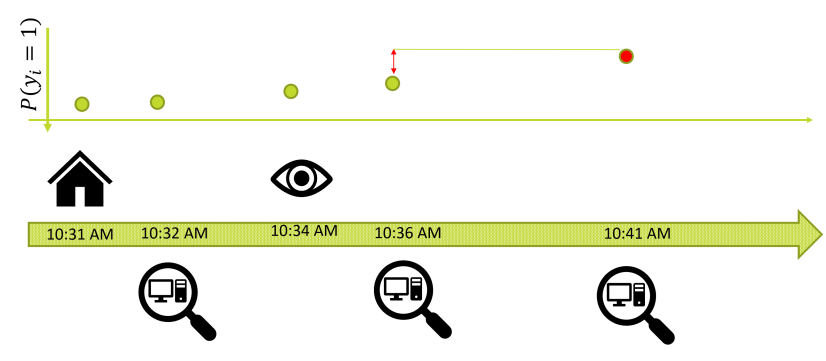

Figure 4: $L\left(e_{j}^{i^{*}}\right)$ : Difference in propensities in the counterfactual.

2.2.3 Critical events for a consumer segment level. : While $Q\left(e_{j}^{i}\right)$ quantifies the criticality of an event at a consumer level, a marketer may be interested in understanding the criticality at a consumer segment level. For instance, say an e-commerce firm recently introduced the "Product Reviews" option on their website. Naturally, a marketer would be interested in understanding if the reviews are helping the consumers in their purchase decisions. To accomodate such inferences, the proposed $Q\left(e_{j}^{i}\right)$ is extended to a consumer segment level by setting up the following hypothesis testing framework. For an event $e_{j}$, we consider all the consumers with $e_{j}$ in their corresponding event streams $E_{j}^{i}$ and set up two one-sided alternative paired t-test to assess the difference in means between $L\left(e_{j}^{i}\right)$ and $L\left(e_{j}^{i^{*}}\right)$, defined in Eq. 1.

$$
\begin{aligned}
& H_{0}: L\left(e_{j}^{i}\right) \leq L\left(e_{j}^{i^{*}}\right) ; \\
& H_{A_{1}}: L\left(e_{j}^{i}\right)>L\left(e_{j}^{i^{*}}\right) ;
\end{aligned}
$$

reject null if event is critical \& helps conversion.

$$
\begin{aligned}
& H_{0}: L\left(e_{j}^{i}\right) \geq L\left(e_{j}^{i^{*}}\right) ; \\
& H_{A_{2}}: L\left(e_{j}^{i}\right)<L\left(e_{j}^{i^{*}}\right) ;
\end{aligned}
$$

reject null if event is critical \& hampers conversion.

As discussed, $L\left(e_{j}\right)$ and $L\left(e_{j}^{*}\right)$ capture the changes of consumer conversion probabilities of an event when the event is present and in the counterfactual when it is absent. Comparing the means while enabling a marketer to identify if a given event is significantly critical across a consumer segment, could also be used to identify a series of critical events. That is, given a consumer segment and a universe of events such as product home, product reviews etc., by applying the hypothesis testing framework for each of the events, a marketer could identify the subset of events that are significantly critical that help the conversion $\left(H_{A_{1}}\right)$ and hamper the conversion $\left(H_{A_{2}}\right)$. We later exemplify the utility of the segment level critical analysis to a marketer for different types of consumer segments in Section 3.

2.2.4 Critical events in marketing funnel transition: Finally, we extend CrEOS's counterfactual analysis framework to identify events that are critical in stage transitions of a marketing funnel. Consumers in an e-commerce website go through a marketing funnel [1]. In an e-commerce website, this funnel can be broadly classified into four different states. 


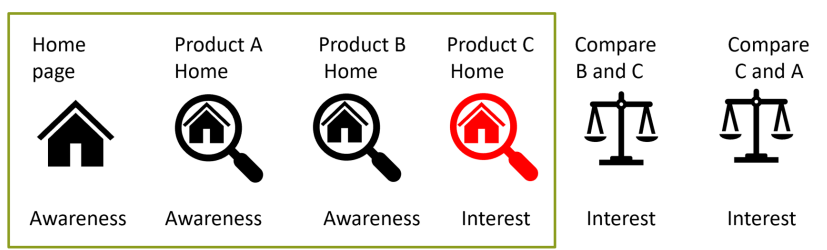

Figure 5: Critical events in marketing funnel transition

(1) Awareness: In this state, a consumer gets aware about the various offerings of an e-commerce firm.

(2) Interest: Once the consumer is aware about the firm, a consumer may shift into an interest stage where she develops an interest in the various product offerings.

(3) Desire: Browsing through the various products, a consumer may desire to purchase a certain product and perform a focused study of the product.

(4) Purchase: Finally, a consumer enters the purchase phase. Here, the consumer purchases the product that she desired, from the website she was made aware of, from one of the products she was interested in.

At each phase, a customer exhibits different browsing behavior [9]. Identifying customer stages enables marketers to perform personalized targeting depending on the stage of a customer. In addition, understanding what events triggered the consumers to move from one stage to another is also crucial. For instance, a consumer in the awareness stage, could move to the interest stage after viewing the stellar customer reviews of a product while another consumer might have triggered due to the product price. Being able to identify this heterogeneity between the consumers would further fine tune the targeting capabilities of a marketer. In our setting, we assume that a marketer has already identified the stages of a consumer and is interested in identifying the critical events that helped them transition between stages.

We exemplify this with an example where we visualize an event sequence of five events in Figure 5. For a consumer $i$ and for each transition of stage (green box in Figure 5), we consider all the events prior to the transition (Home page, Prod.A home, Prod.B home) and the event at the transition (Prod.C home). We estimate the criticality of each of these events $Q\left(e_{j}^{i}\right)$ as described in Eq. 1 . Formally, assume the transition occurred at $e_{t}^{i}$, we compute $Q\left(e_{j}^{i}\right)$ $\forall e_{j}^{i} \in E_{t}^{i} \subset E_{k_{i}}^{i}$. The estimated $Q\left(e_{j}^{i}\right)$ can be further extended to a consumer segment to assess whether an event $e_{j}^{i}$ was critical, identify a series of consumer events in stage transition using the hypothesis testing framework discussed in Eq. 2, 3.

\section{EXPERIMENTS}

We apply the proposed methodology on a snapshot of clickstream event data of a large US based e-commerce firm. We break down the event stream into multiple consumer sessions whenever there is an inactivity of thirty minutes between two events. The sessionization of clickstream data is a standard pre-processing technique in clickstream analysis $[12,13]$. Next, we remove all sessions with less than five click events. The final sample comprised of 10,234
Table 1: Early detection performance - single variable LSTM.

\begin{tabular}{c|c|c|c|c}
\hline $\begin{array}{c}\% \\
\text { of click } \\
\text { events }\end{array}$ & Precision & Recall & $\begin{array}{c}\text { F-1 } \\
\text { Score }\end{array}$ & AUC \\
\hline $25 \%$ & 0.57 & 0.72 & 0.64 & 0.63 \\
\hline $50 \%$ & 0.65 & 0.67 & 0.66 & 0.69 \\
\hline $75 \%$ & 0.70 & 0.72 & 0.71 & 0.74 \\
\hline $100 \%$ & 0.72 & 0.87 & 0.79 & 0.79 \\
\hline \hline
\end{tabular}

consumer sessions with around $4 \%$ purchasers. In total, we had a total of 99 click event types (Guest Sign in, Browse by Category etc.) in our data set.

\subsection{Sequential model}

We model the $P\left(y_{k_{i}}^{i} \mid\left\{E_{k_{i}}^{i}, C_{k_{i}}^{i}\right\}\right)$ as a binary classification problem. To train the LSTM, we first embed the categorical features in $\left\{E_{k_{i}}^{i}, C_{k_{i}}^{i}\right\}$ and concatenate them with the numerical features. Multiple fully connected dense layers are added to the output of the LSTM to estimate the probability of conversion of a given event and context stream. Further, to tackle the class imbalance which is natural in an e-commerce setting ${ }^{2}$, we experiment with different sample weights assigned based on the conversion outcome. Finally, we perform a five-fold cross validation and tune three parameters number of number of dense layers, optimizer and sample weights to maximize precision and estimate $P\left(y_{k_{i}}^{i} \mid\left\{E_{k_{i}}^{i}, C_{k_{i}}^{i}\right\}\right)$.

\subsection{CrEOS for early detection:}

In Table 1,2, we compare CrEOS's multi-variable context enriched LSTM to a single-variable LSTM proposed in [12]. Specifically, we compare the early detection performance of the learned models on test data. This is done to mimic a marketer who would be interested in knowing if a consumer is going to convert as early as possible in a click session. We truncate each session in the test set at 25\%,50\% and $75 \%$ and report the precision, recall, F-1 scores and AUC for the two models. We observe that CrEOS's multi-variable LSTM is at par with the LSTM learnt just based on the click events at $25 \%$ and outperforms the baseline in terms of AUC as more of the sequence is revealed. Similar observations can be made from the precision-recall curve visualized in Figure 6 where the proposed conversion model outperforms the single variable LSTM. This empirically justifies the addition of context to the event stream discussed in Section 2.2.1 and visualized in Figure 2.

\subsection{CrEOS for consumer segments}

In Table 3, we discuss the critical events identified by CrEOS's hypothesis testing framework for different consumer segments. We handcraft four different consumer segments to mimic a marketer and identify the subset of events that help or hamper the online purchase from the 99 events in the data set. The four segments were handcrafted based on the browsing behaviour of the consumers in the snapshot.

\footnotetext{
${ }^{2}$ On an e-commerce website, for every 100 consumers only 2-3 end up converting.
} 
Table 2: Early detection performance of CrEOS

\begin{tabular}{c|c|c|c|c}
\hline $\begin{array}{c}\text { \% } \\
\text { of click } \\
\text { events }\end{array}$ & Precision & Recall & $\begin{array}{c}\text { F-1 } \\
\text { Score }\end{array}$ & AUC \\
\hline $25 \%$ & 0.62 & 0.37 & 0.46 & 0.62 \\
\hline $50 \%$ & 0.73 & 0.45 & 0.56 & 0.73 \\
\hline $75 \%$ & 0.80 & 0.56 & 0.66 & 0.79 \\
\hline $100 \%$ & 0.86 & 0.82 & 0.84 & 0.89 \\
\hline \hline
\end{tabular}

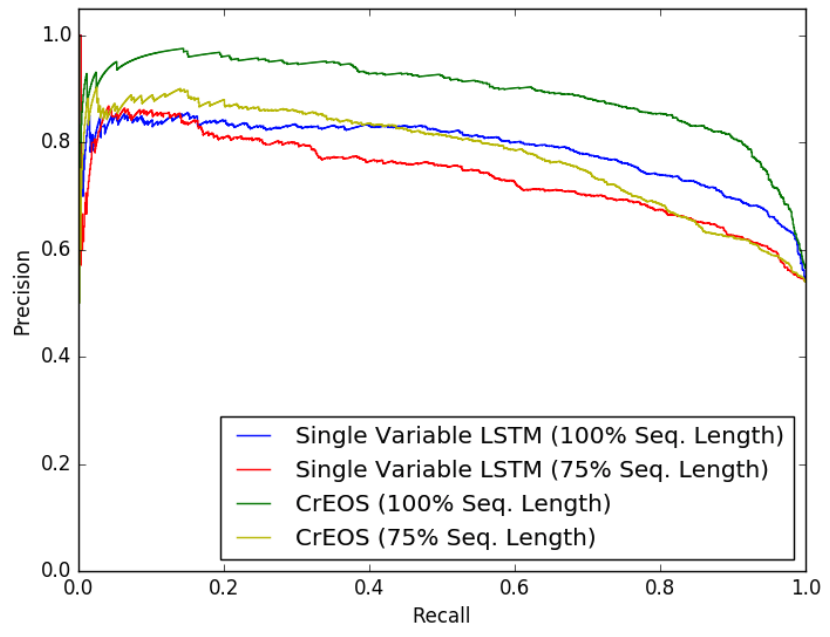

Figure 6: Precision-Recall curves

Table 3: CrEOS for consumer segments

\begin{tabular}{|c|c|c|}
\hline $\begin{array}{c}\text { Consumer } \\
\text { Segment }\end{array}$ & $\begin{array}{c}\text { Help } \\
\text { online } \\
\text { purchase }\end{array}$ & $\begin{array}{c}\text { Hamper } \\
\text { online } \\
\text { purchase }\end{array}$ \\
\hline Product hoARdERS & $\begin{array}{l}\text { Product Compare, } \\
\text { Product Search }\end{array}$ & $\begin{array}{l}\text { Store Locator, } \\
\text { Home Page }\end{array}$ \\
\hline OFFER GRABBERS & Product Reviews & $\begin{array}{l}\text { Store locator, } \\
\text { Home page, } \\
\text { Guest Sign In. }\end{array}$ \\
\hline CART ADDITIONS & $\begin{array}{l}\text { Product Compare, } \\
\text { Rewards Page, } \\
\text { Product Reviews }\end{array}$ & $\begin{array}{c}\text { Product Questions, } \\
\text { Deals page, } \\
\text { Product Search. }\end{array}$ \\
\hline Time Spenders & $\begin{array}{c}\text { Browse by Category, } \\
\text { Product Questions }\end{array}$ & $\begin{array}{l}\text { Product Reviews, } \\
\text { Product Search, } \\
\text { Chat with an Agent, } \\
\text { Rewards page }\end{array}$ \\
\hline
\end{tabular}

(1) Product Hoarders - consumers who have added at least five products in the cart during one session.

(2) OfFER GRABbers - consumers that have applied an offer at least once in a session.

(3) CART ADDitions - consumers that have added at least three items to their cart in a session.
(4) Time SPENDERS - consumers who spend more time than an average consumer (belong to the $80-100$ percentile of the consumer time spent distribution).

In Table 3, we observe that the events "Product Compare" and "Product Search" help the purchase propensity of Product HoARders while "Store Locator" and "Home Page" hamper it. This indicates that if PRODUCT HoARDERs search and compare different products, they are more likely to make a purchase online than those who do not. Also, if a Product HoArder browse for the "Store Locator" page, they are likely to not make the purchase online but rather just get their research done on the website before heading to the store. Drawing a similar inference, OFFER GRABBERS are more likely to make a purchase if they are looking at the product reviews and are deterred if they see a "Guest Sign In" page on the website. Also, a consumer in CART ADDITIONS is more likely to purchase if they compare multiple products and browse through product reviews. As expected, we notice a majority of landing pages such as Home Page, Deals page, Product Promo appearing in the events that hamper the propensity of consumers.

\subsection{CrEOS for marketing funnel transitions}

In Table 4, we tabulate the segment level critical events for different transitions in the marketing funnel. In CrEOS, as stated earlier, we assume that a consumer stage is given prior to identifying the critical events in stage transitions. To assign the stages, we use the distribution of the estimated conversion probabilities across all the consumers to compute corresponding quantile value for three different quantiles - 50\% (Awareness), 75\% (Interest), 96\% (Desire) and Purchase. The assumption here is that close to half of the users in each stage progress to the next stage. Depending on the corresponding purchase probabilities, each event in a consumer session is then assigned a customer stage - Awareness - (0 - 50\% quantile), Interest (50 - 75\% quantile) and Desire (75 - 87.5\% quantile). Hence a consumer who has a conversion probability at a certain click event less than $50 \%$ of the consumers is assigned an Awareness stage and so on.

In Table 4, we observe that for a consumer in the awareness stage, "Product Search", "Browse by category" events which demonstrate a more focused search by a consumer are critical in their movement to the Interest stage. For a consumer in the desire stage, events such as "Product reviews", "Questions" move them toward the purchase stage indicating a product specific quality assessment by the consumer before deciding to purchase (Action stage). Further, generic landing web pages such as Home Page, Deals page, Account Page hamper the transition to the next stage in the marketing funnel.

\section{RELATED WORK}

Modeling conversion behaviour in event streams is an active research area. Traditionally, the methods relied on hand crafted feature engineering from the event streams [4, 13]. [13] model click behaviour in click streams by constructing features based on frequency, time spent on visited web pages and learns a metric to compute similarity between purchasers. [4] builds a scalable heuristic to predict conversion in display advertising using a logistic regression. More recently, the trend has shifted to the deployment of deep learning techniques to model consumer online behaviour. RNNs have 
Table 4: CrEOS for marketing funnel transitions

\begin{tabular}{|c|c|c|}
\hline $\begin{array}{c}\text { Stage } \\
\text { transition }\end{array}$ & $\begin{array}{c}\text { Help } \\
\text { online } \\
\text { purchase }\end{array}$ & $\begin{array}{l}\text { Hamper } \\
\text { online } \\
\text { purchase }\end{array}$ \\
\hline $\begin{array}{l}\text { Awareness to } \\
\text { Interest }\end{array}$ & $\begin{array}{c}\text { Browse by Category, } \\
\text { Product Search, } \\
\text { Product Questions }\end{array}$ & $\begin{array}{l}\text { Guest Sign in, } \\
\text { Home Page, } \\
\text { Product Deals }\end{array}$ \\
\hline $\begin{array}{l}\text { Interest to } \\
\text { Desire }\end{array}$ & $\begin{array}{l}\text { Product Questions, } \\
\text { Product Reviews, } \\
\text { Store locator, } \\
\text { Tech Support, } \\
\text { Sign in }\end{array}$ & $\begin{array}{c}\text { Global Deals, } \\
\text { Home Page, } \\
\text { Product Search, } \\
\text { Browse by Category, } \\
\text { Sign Out }\end{array}$ \\
\hline Desire to Action & $\begin{array}{l}\text { Product Questions, } \\
\text { Product Reviews, } \\
\text { Product Compare, } \\
\text { Store locator, } \\
\text { Chat with } \\
\text { an Agent. }\end{array}$ & $\begin{array}{l}\text { Browse by Category, } \\
\text { Product Search, } \\
\text { Sign Out, } \\
\text { Account Home, } \\
\text { Home Page }\end{array}$ \\
\hline
\end{tabular}

have been studied and successfully applied to predict click rates for ads on search result pages [18], click through rate prediction [19], proxy survey ratings [15], item recommendations in a consumer session [8], consumer conversion prediction [12,14]. Both $[12,14]$ employ a single variable LSTM to model conversion behavior. [12] interprets the contribution of each event to the conversion propensity by linking the LSTM cells to the predicted conversion probabilities. [14] adds an attention layer [10] to the LSTM to explain the conversion predictions. The proposed sequential model in CrEOS improves upon these studies by enriching the event stream with contextual features. Attention mechanism in multi-variable LSTMs remain relatively unexplored [7, 17]. [17] shows that the contribution of individual variables in multi-variable LSTM is intractable to distinguish through the sequence of hidden states. [7] jointly learn network parameters associated with variable and temporal importance in predicting the target variable. In CrEOS, we perform a post hoc analysis leveraging concepts from econometrics on the learned multi-variable LSTM to identify critical events that either help or hamper a consumer conversion. We extend the critical event identification to a consumer segment level by setting up a hypothesis testing framework.

\section{CONCLUSION}

In this work, we propose CrEOS, an end to end framework to predict a consumer's conversion propensity and identify critical events that either help or hamper the propensity in an online session. Our method has the following advantages for a marketer compared to the current academic literature.

(1) Conversion propensity - Given a user's event stream, CrEOS accurately predicts the conversion propensity after each click event when deployed on a clickstream data set from a large ecommerce based US firm. This is enabled by augmenting the event stream with contextual features and minimal feature engineering. This accurate dynamic conversion propensity would help a marketer perform real time targeting.
(2) Critical event identification - CrEOS enables a marketer to automatically mine through several events triggered during a customer visit on an e-commerce platform to identify and quantify their marginal contribution towards conversion propensity. CrEOS also identifies critical events for a consumer segment that a marketer is interested in and their transitions between different stages of a marketing funnel. We discuss the insights a marketer could gain from the identification by considering multiple use cases of a marketer.

\section{REFERENCES}

[1] Vibhanshu Abhishek, Peter Fader, and Kartik Hosanagar. 2012. Media exposure through the funnel: A model of multi-stage attribution. Available at SSRN 2158421 (2012).

[2] Richard Ernest Bellman and Kenneth L Cooke. 1963. Differential-difference equations. (1963).

[3] Randolph E Bucklin and Catarina Sismeiro. 2003. A model of web site browsing behavior estimated on clickstream data. Fournal of marketing research 40, 3 (2003), 249-267.

[4] Olivier Chapelle, Eren Manavoglu, and Romer Rosales. 2015. Simple and scalable response prediction for display advertising. ACM Transactions on Intelligent Systems and Technology (TIST) 5, 4 (2015), 61.

[5] Min Chen, Shiwen Mao, and Yunhao Liu. 2014. Big data: A survey. Mobile networks and applications 19, 2 (2014), 171-209.

[6] Anindya Ghose. 2017. TAP: Unlocking the mobile economy. MIT Press.

[7] Tian Guo, Tao Lin, and Nino Antulov-Fantulin. 2019. Exploring Interpretable LSTM Neural Networks over Multi-Variable Data. arXiv preprint arXiv:1905.12034 (2019).

[8] Balázs Hidasi, Alexandros Karatzoglou, Linas Baltrunas, and Domonkos Tikk. 2015. Session-based recommendations with recurrent neural networks. arXiv preprint arXiv:1511.06939 (2015)

[9] John A Howard and Jagdish N Sheth. 1969. The theory of buyer behavior. New York 63 (1969).

[10] Andrej Karpathy, Justin Johnson, and Li Fei-Fei. 2015. Visualizing and understanding recurrent networks. arXiv preprint arXiv:1506.02078 (2015).

[11] Mandy Korpusik, Shigeyuki Sakaki, Francine Chen, and Yan-Ying Chen. 2016. Recurrent Neural Networks for Customer Purchase Prediction on Twitter. CBRecSys@RecSys 1673 (2016), 47-50.

[12] Tobias Lang and Matthias Rettenmeier. 2017. Understanding consumer behavior with recurrent neural networks. In Workshop on Machine Learning Methods for Recommender Systems.

[13] Deepak Pai, Abhijit Sharang, Meghanath Macha Yadagiri, and Shradha Agrawal. 2014. Modelling visit similarity using click-stream data: A supervised approach. In International Conference on Web Information Systems Engineering. Springer, 135-145.

[14] Balaraman Ravindran et al. 2018. A neural attention based approach for clickstream mining. In Proceedings of the ACM India foint International Conference on Data Science and Management of Data. ACM, 118-127.

[15] Atanu R Sinha, Deepali Jain, Nikhil Sheoran, Sopan Khosla, and Reshmi Sasidharan. 2019. Surveys Without Questions: A Reinforcement Learning Approach. (2019).

[16] Shuba Srinivasan, Oliver J Rutz, and Koen Pauwels. 2016. Paths to and off purchase: quantifying the impact of traditional marketing and online consumer activity. Fournal of the Academy of Marketing Science 44, 4 (2016), 440-453.

[17] Liheng Zhang, Charu Aggarwal, and Guo-Jun Qi. 2017. Stock price prediction via discovering multi-frequency trading patterns. In Proceedings of the $23 \mathrm{rd} \mathrm{ACM}$ SIGKDD international conference on knowledge discovery and data mining. ACM, 2141-2149.

[18] Yuyu Zhang, Hanjun Dai, Chang Xu, Jun Feng, Taifeng Wang, Jiang Bian, Bin Wang, and Tie-Yan Liu. 2014. Sequential click prediction for sponsored search with recurrent neural networks. In Twenty-Eighth AAAI Conference on Artificial Intelligence.

[19] Guorui Zhou, Xiaoqiang Zhu, Chenru Song, Ying Fan, Han Zhu, Xiao Ma, Yanghui Yan, Junqi Jin, Han Li, and Kun Gai. 2018. Deep interest network for click-through rate prediction. In Proceedings of the 24th ACM SIGKDD International Conference on Knowledge Discovery \& Data Mining. ACM, 1059-1068. 